\title{
Morphométrie des adultes de Diastocera trifasciata (Fabricius, 1775) (Coleoptera : Cerambycidae), ciseleur des branches d'anacardiers dans la région du Gbêkê (Centre de la Côte d'Ivoire)
}

\author{
OUALI N'GORAN San-Whouly Mauricette, KOFFI Kouassi Misler, AKESSÉ Ettien Narcice et \\ EHOUNOU Prisca Gnanda \\ Université Félix HOUPHOUET-BOIGNY / UFR Biosciences/ Laboratoire des milieux Naturels et de Conservation de \\ la Biodiversité, Côte d'Ivoire \\ Auteur Correspondant : OUALI N'GORAN San-Whouly Mauricette, Email : ngoransw@yahoo.fr / Tél : (225) 0740 \\ 5705
}

Original submitted in on 23rd March 2020. Published online at www.m.elewa.org/journals/ on 30th April 2020

https://doi.org/10.35759/JABs.148.7

\section{RESUME}

Objectif : la présente étude a été conduite afin de connaître les caractères morphométriques des adultes de Diastocera trifasciata pour faciliter son identification dans les vergers d'anacardier de Côte d'Ivoire.

Méthodologie et résultats : En octobre 2018, des prospections dans les vergers d'anacardier à Brobo au Centre de la Côte d'Ivoire ont permis de collecter 42 mâles et 42 femelles. Ces insectes ont été asphyxiés à l'aide d'éther éthylique avant d'être disséqués. Les mesures de la longueur du corps, de la tête, des antennes, des pièces buccales, du pronotum, des ailes, des pattes et du dernier sternite abdominal ont été prises avec un pied à coulisse. Les résultats révèlent qu'il n'y a pas de différence au niveau de la taille du corps, des pièces buccales, des pattes entre les deux sexes. Par contre, un dimorphisme sexuel a été observé par la taille des antennes et du dernier sternite abdominal. Les antennes mesurent 56,66 $\pm 5,86$ $\mathrm{mm}$ chez les mâles contre 49,17 $\pm 4,81 \mathrm{~mm}$ chez les femelles. Le dernier sternite abdominal mesure 2,87 $\pm 0,39 \mathrm{~mm}$ de long chez les mâles contre $4,03 \pm 0,26 \mathrm{~mm}$ chez les femelles où il est marqué par un sillon longitudinal.

Conclusion et applications : Cette étude a permis de connaître les caractères morphométriques des adultes mâles et femelles de Diastocera trifasciata. Les résultats de cette étude constituent une base de données pour une identification précise de $D$. trifasciata. Cela permettra de mener une lutte ciblée contre elle et préserver la diversité de l'entomofaune utile aux vergers anacardiers.

Mots clés : Morphométrie, Diastocera trifasciata, anacardiers, dimorphisme sexuel, Côte d'Ivoire.

\section{INTRODUCTION}

L'anacardier est une plante pérenne qui occupe depuis quelques années, une place importante dans l'agriculture ivoirienne. Troisième plus important produit d'exportation de la Côte d'Ivoire après le cacao et le caoutchouc en 2010 (Koné,
2010 ; Lebally et al., 2012); cette culture occupe aujourd'hui la deuxième place après la cacaoculture. Depuis 2015, la Côte d'Ivoire est le premier producteur et exportateur mondial de noix de cajou brutes avec une production estimée à 
environ 700000 tonnes/an, soit $25 \%$ de la production mondiale (CCA, 2016 ; Diop, 2016). Malgré ces performances remarquables, le rendement des vergers ivoiriens $(350$ à $500 \mathrm{~kg} / \mathrm{h}$ ) demeure en dessous du rendement standard international compris entre 1000 à $5000 \mathrm{~kg} / \mathrm{ha}$ (CCA, 2016 ; Djaha et al., 2017). Les causes de ce faible rendement sont la persistance des problèmes phytosanitaires, notamment les attaques d'insectes ravageurs (Viana et al., 2007). En Côte d'lvoire, parmi ces insectes ravageurs, l'adulte de Diastocera trifasciata, espèce anciennement appelée Analeptes trifasciata, est un ravageur majeur de l'anacardier (Akessé et al., 2015; N'Dépo et al., 2017). Ces adultes coupent les branches d'anacardier et peuvent occasionner de sérieuses pertes de rendement des noix de cajou de plus de 55\% (Adeigbe et al., 2015; Akessé et al., 2015). Les dégâts de cet insecte ont été signalés la première fois en Côte d'Ivoire en

\section{MATÉRIEL ET MÉTHODES}

Site d'étude: Les études ont été réalisées de Septembre à Novembre 2018 à Brobo (Longitude : $004^{\circ} 49^{\prime} 29^{\prime \prime}$, latitude: $07^{\circ} 38^{\prime} 18^{\prime \prime}$, altitude $246 \mathrm{~m}$ ), localité située au Centre de la Côte d'Ivoire, à $20 \mathrm{Km}$ de la ville Bouaké sur l'axe Bouaké-M'Bahiakro. Elle appartient à la zone soudano-guinéenne caractérisée par un climat de type équatorial de transition avec des précipitations annuelles variant de $1200 \mathrm{~mm}$ à 1500 $\mathrm{mm}$. La température moyenne varie de $20^{\circ} \mathrm{C}$ à 27,5 ${ }^{\circ} \mathrm{C}$. L'humidité relative est comprise entre $57 \%$ et $85 \%$ (FAO, 2005).

Collecte des insectes : Les insectes ont été capturés à la main ou à l'aide d'une perche sur les anacardiers dans trois vergers attaqués par l'espèce. La présence de sciure de bois sur les feuilles sèches au sol et de branches annelées accrochées sur les anacardiers ont été les indices de repérage des adultes de Diastocera trifasciata sur les anacardiers. Après leur capture, les insectes sont introduits dans les boîtes de collecte préalablement perforées pour permettre leur aération et convoyés au laboratoire. Les insectes sont ensuite asphyxiés à l'aide d'éther éthylique pour les différentes manipulations.

Description morphologique des insectes: Les différentes régions du corps de l'insecte ont été décrites. II s'agit des antennes et des pièces buccales au niveau de la tête ; du pronotum, des ailes
1964 dans les champs de Kokondekro par Brunck et Fabre (1970). Malheureusement, très peu de travaux ont été consacrés à cette espèce du fait du manque d'enjeu économique de l'anacardier pour les producteurs de Côte d'Ivoire (Hammed et al., 2008). Les études récentes ont porté sur les dégâts, la biologie et la fluctuation de l'espèce sans toutefois décrire les caractères morphologiques de l'insecte (Kra et al., 2017 ; Akessé et Ouali N'Goran, 2018 ; Akessé et al., 2018). Par ailleurs, N'Dépo et al. (2017) ont signalé la présence d'autres espèces de Cerambycidae morphologiquement semblables à $D$. trifasciata. Face à ce constat, il s'avère nécessaire de reconnaitre avec précision ce ravageur pour une lutte ciblée et efficace. L'objectif de cette étude est donc de connaître les caractères morphométriques des adultes de Diastocera trifasciata afin de contribuer à une meilleure identification.

et des pattes au niveau du thorax et du dernier sternite au niveau de l'abdomen de l'espèce.

Mensurations des insectes (Figure 1): Les insectes sont ensuite disposés dans un bac à dissection pour être disséqués et mesurés. Les mensurations ont été réalisées à l'aide d'un pied à coulisse et ont concerné :

- la longueur du corps : du point supérieur de la tête à l'extrémité abdominale ;

- la largeur du corps : à la base des élytres ;

- la longueur de la tête : dans le sens du prolongement des pièces buccales, du sommet de la tête à l'extrémité des pièces buccales ;

- la largeur de la tête : au niveau des yeux composés; la longueur totale de l'antenne et de chaque article antennaire ;

- la longueur et la largeur des pièces buccales (labre, mandibules, maxilles et labium);

- la longueur et la largeur du pronotum ;

- la longueur de l'élytre : du point d'insertion de l'élytre à l'apex ;

La largeur de l'aile membraneuse : à la base des ailes ; la longueur des pattes antérieures, médianes et postérieures et de celle de chaque article des pattes concernées; la longueur et la largeur du dernier sternite abdominal.

Pour une meilleure comparaison des moyennes entre mâles et femelles, le même nombre d'individus a été 
retenu pour les deux sexes. Ainsi, parmi les insectes collectés, c'est un échantillon de 84 individus à savoir 42 mâles et 42 femelles qui ont servi à la prise des mesures. II s'agit des insectes n'ayant subi aucun dommage physique lors des captures et du transport au laboratoire.
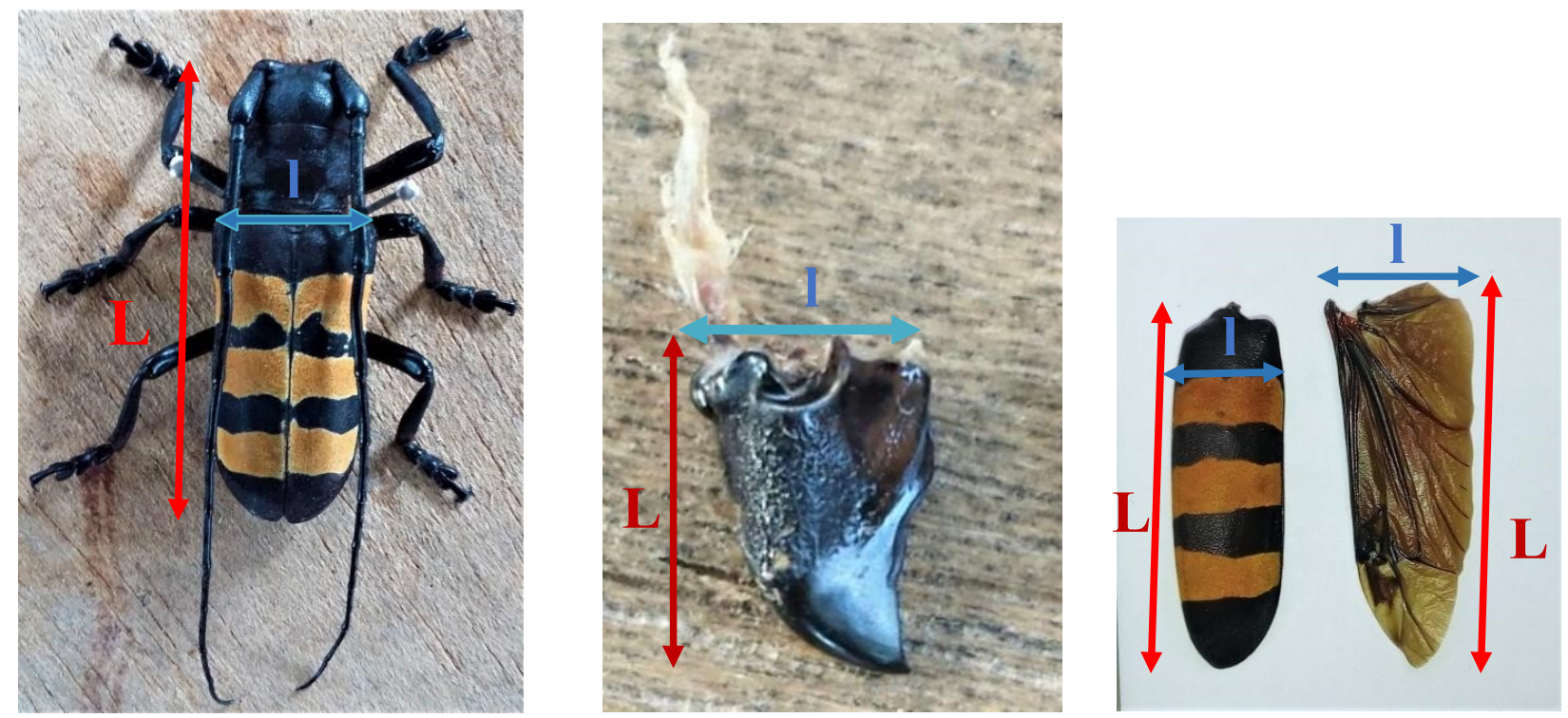

Figure 1 : Quelques mensurations faites sur Diastocera trifasciata

$$
\mathrm{L}=\text { longueur } ; \mathrm{I} \text { = largeur }
$$

Analyses statistiques : Les données obtenues ont été soumises à une analyse de variance (ANOVA) au seuil de $5 \%$ à l'aide du logiciel Statistica version 7.1. En cas

\section{RÉSULTATS}

La description des formes et des tailles des différents organes étudiés s'est fait simultanément pour mieux apprécier les ressemblances et les différences liées au sexe.

Description générale des adultes de Diastocera trifasciata: Chez l'espèce Diastocera trifasciata, certains caractères morphologiques sont semblables chez le mâle et la femelle. Diastocera trifasciata est un longicorne au corps robuste, très sclérifié dont la tête, les antennes, le thorax, les pattes et l'abdomen sont de couleur noir. Les antennes chez cette espèce sont de différence significative, le test de Newman-Keuls a permis de séparer les moyennes des différentes mensurations.

longues et filiformes de couleurs noires. Elles se composent de 11 articles. De la base de l'antenne vers son extrémité, on distingue le scape, le pédicelle et neuf flagellomères. Les mandibules sont de forme triangulaire, coriaces, très sclérifiées, rugueuses sur la partie antérieure et lisse sur la partie postérieure. La surface coupante, pointue et crochue se trouve sur la partie postérieure. Les élytres possèdent quatre bandes noires continues ou non et trois bandes jaunes ou jaune-orangés à disposition alternée (Figure 2). 

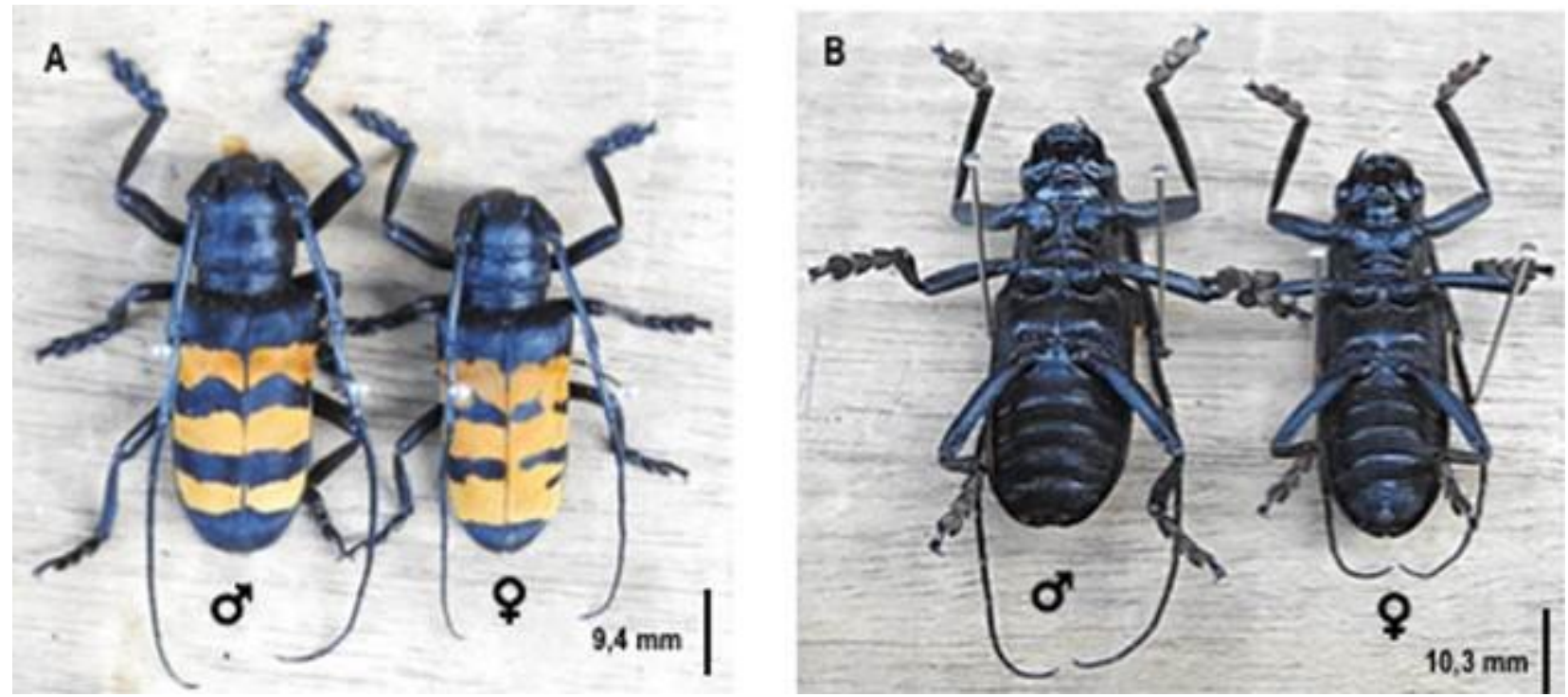

Figure 2 : Adultes de Diastocera trifasciata, $\mathrm{A}$ : vue dorsale et $\mathrm{B}$ : vue ventrale

\section{Mensurations de D. trifasciata}

Corps: La longueur du corps des mâles et des femelles de $D$. trifasciata est respectivement de 40,17 \pm $3,28 \mathrm{~mm}$ et $41,40 \pm 3,19 \mathrm{~mm}$. La tête de l'insecte mesure $12,68 \pm 1,41 \mathrm{~mm}$ de long et $9,20 \pm 1,10 \mathrm{~mm}$ de large chez le mâle et $12,68 \pm 1,77 \mathrm{~mm}$ de long et 9,57 $\pm 1,16 \mathrm{~mm}$ de large chez la femelle. Aucune différence significative n'a été révélée par les résultats de I'ANOVA (Tableau 1). Le dernier sternite abdominal est plus court chez le mâle avec une longueur moyenne de $2,87 \pm 0,39 \mathrm{~mm}$ contre $4,03 \pm 0,26 \mathrm{~mm}$ chez la femelle. Par ailleurs, un sillon longitudinal est présent au niveau du dernier sternite abdominal de la femelle (Figure 3).

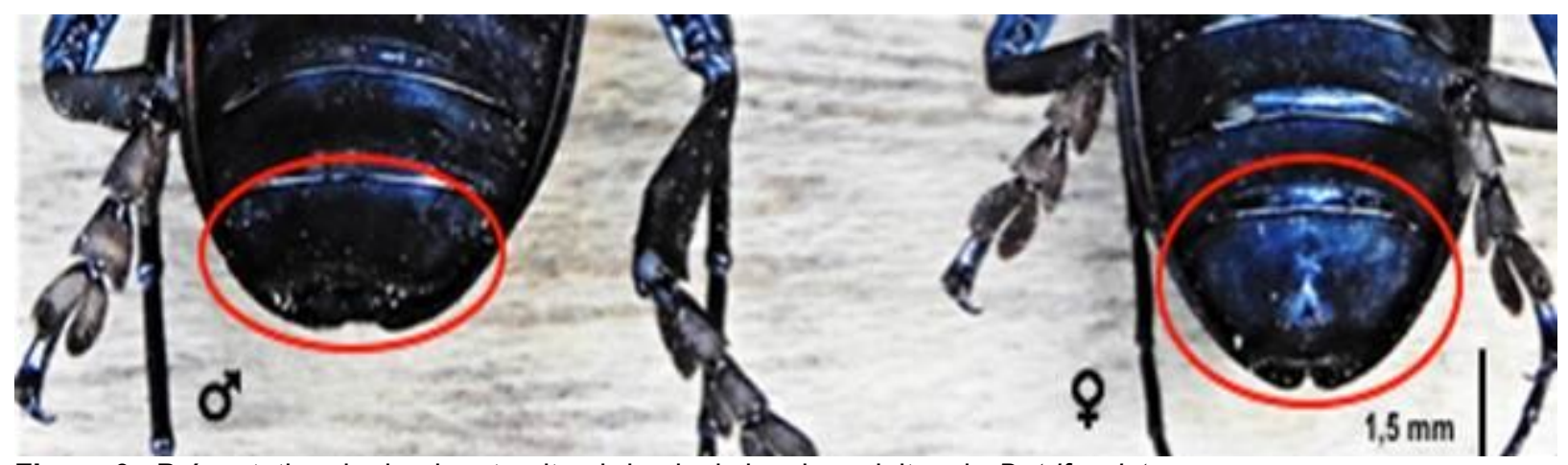

Figure 3 : Présentation du dernier sternite abdominal chez les adultes de $D$. trifasciata

Ailes : Chez les mâles, l'élytre mesure 29,97 $\pm 2,28$ $\mathrm{mm}$ de long contre $31,12 \pm 2,19 \mathrm{~mm}$ chez les femelles, soit $1,15 \mathrm{~mm}$ de différence $(F=5,61 ; d d l=1 ; P=$
0,02). Au niveau des ailes membraneuses aucune différence significative n'a été enregistrée entre les mâles et les femelles (Tableau 1). 
Tableau 1: Morphométrie du corps des adultes de Diastocera trifasciata suivant le sexe

\begin{tabular}{lll}
\hline \multirow{2}{*}{ Caractères } & \multicolumn{1}{l}{ Moyenne \pm écart-type } \\
\cline { 2 - 3 } & Longueur (mm) & Largeur (mm) \\
\hline $\begin{array}{l}\text { Corps entier } \\
\text { Mâle }\end{array}$ & $40,17 \pm 3,28 \mathrm{a}$ & $13,77 \pm 1,30 \mathrm{a}$ \\
$\quad$ Femelle & $41,40 \pm 3,19 \mathrm{a}$ & $14,16 \pm 1,71 \mathrm{a}$ \\
Tête & $12,68 \pm 1,41 \mathrm{a}$ & \\
$\quad$ Mâle & $12,68 \pm 1,77 \mathrm{a}$ & $9,20 \pm 1,10 \mathrm{a}$ \\
$\quad$ Femelle & $7,13 \pm 0,95 \mathrm{a}$ & $9,57 \pm 1,16 \mathrm{a}$ \\
Pronotum & $6,99 \pm 0,46 \mathrm{a}$ & $9,67 \pm 1,4 \mathrm{a}$ \\
$\quad$ Mâle & & $9,69 \pm 0,64 \mathrm{a}$ \\
$\quad$ Femelle & $29,97 \pm 2,28 \mathrm{a}$ & $9,22 \pm 0,74 \mathrm{a}$ \\
Elytre & $31,12 \pm 2,19 \mathrm{~b}$ & $9,44 \pm 0,68 \mathrm{a}$ \\
$\quad$ Mâle & $34,51 \pm 2,56 \mathrm{a}$ & $13,31 \pm 1,31 \mathrm{a}$ \\
$\quad$ Femelle & $35,12 \pm 2,37 \mathrm{a}$ & $13,12 \pm 1,28 \mathrm{a}$ \\
Aile membraneuse & & \\
$\quad$ Mâle & Femelle &
\end{tabular}

Chaque variable dans une même colonne, les valeurs moyennes portant les mêmes lettres, ne sont pas significativement différentes $(p>0,05)$.

Pièces buccales: Au niveau des pièces buccales, aucune différence significative n'a été observée entre les mâles et les femelles. Les longueurs et les largeurs

du labre, des mandibules, des maxilles et du labium chez le mâle et chez la femelle sont statistiquement égales $(p>0,05)$ (Tableau 2).

Tableau 2 : Morphométrie des pièces buccales des adultes de $D$. trifasciata

\begin{tabular}{lll}
\hline Caractères & Moyenne \pm écart-type & \\
\cline { 2 - 3 } & Longueur $(\mathbf{m m})$ & Largeur $(\mathbf{m m})$ \\
\hline Labre & $4,75 \pm 0,65 \mathrm{a}$ & $3,86 \pm 0,45 \mathrm{a}$ \\
Mâle & $4,82 \pm 0,65 \mathrm{a}$ & $4,01 \pm 0,52 \mathrm{a}$ \\
$\quad$ Femelle & & \\
Mandibule & $5,39 \pm 0,61 \mathrm{a}$ & $3,71 \pm 0,52 \mathrm{a}$ \\
$\quad$ Mâle & $5,39 \pm 0,48 \mathrm{a}$ & $3,61 \pm 0,39 \mathrm{a}$ \\
$\quad$ Femelle & & \\
Maxille & $6,32 \pm 0,68 \mathrm{a}$ & $2,35 \pm 0,45 \mathrm{a}$ \\
$\quad$ Mâle & $6,06 \pm 0,65 \mathrm{a}$ & $2,42 \pm 0,42 \mathrm{a}$ \\
Femelle & & \\
Labium & $5,50 \pm 0,63 \mathrm{a}$ & $2,29 \pm 0,46 \mathrm{a}$ \\
Mâle & $5,52 \pm 0,59 \mathrm{a}$ & $2,28 \pm 0,46 \mathrm{a}$ \\
Femelle & &
\end{tabular}
différentes $(p>0,05)$.

Antennes: La taille des antennes de l'insecte est comprise entre $35,37 \mathrm{~mm}$ et $67,11 \mathrm{~mm}$. Leur longueur moyenne est $52,92 \pm 6,53 \mathrm{~mm}$. Elles sont donc plus longues que le corps de l'insecte. Elles se composent de onze articles dont le scape, le pédicelle et neuf flagellomères formant le flagelle. Chez les mâles, elles mesurent $56,66 \pm 5,86 \mathrm{~mm}$ soit 1,41 fois la longueur du corps et chez les femelles, elles mesurent 49,17 $\pm 4,81$ $\mathrm{mm}$ soit 1,18 fois la longueur du corps. La différence entre la longueur des antennes est très significative entre les mâles et les femelles ( $F=40,91$; ddl $=1 ; P=$ 0,00) (Tableau 3).

Pattes: Au niveau des trois paires de pattes, aucune différence significative n'a été relevée entre les mâles et les femelles (Tableau 4). 
Tableau 3 : Morphométrie de l'antenne des adultes de Diastocera trifasciata

\begin{tabular}{lll}
\hline \multirow{2}{*}{ Segment $(\mathbf{m m})$} & \multicolumn{2}{c}{ Moyenne \pm écart-type } \\
\cline { 2 - 3 } & Mâle & Femelle \\
\hline Scape & $4,82 \pm 0,56 \mathrm{a}$ & $4,47 \pm 0,48 \mathrm{~b}$ \\
Pédicelle & $1,61 \pm 0,27 \mathrm{a}$ & $1,47 \pm 0,25 \mathrm{~b}$ \\
A1 & $10,58 \pm 1,23 \mathrm{a}$ & $9,79 \pm 0,91 \mathrm{~b}$ \\
A2 & $8,50 \pm 0,96 \mathrm{a}$ & $7,35 \pm 0,76 \mathrm{~b}$ \\
A3 & $5,79 \pm 0,60 \mathrm{a}$ & $4,92 \pm 0,48 \mathrm{~b}$ \\
A4 & $5,06 \pm 0,54 \mathrm{a}$ & $4,18 \pm 0,43 \mathrm{~b}$ \\
A5 & $4,69 \pm 0,50 \mathrm{a}$ & $3,90 \pm 0,41 \mathrm{~b}$ \\
A6 & $4,33 \pm 0,47 \mathrm{a}$ & $3,48 \pm 0,39 \mathrm{~b}$ \\
A7 & $4,10 \pm 0,49 \mathrm{a}$ & $3,35 \pm 0,41 \mathrm{~b}$ \\
A8 & $3,85 \pm 0,48 \mathrm{a}$ & $3,15 \pm 4,40 \mathrm{~b}$ \\
A9 & $3,83 \pm 0,50 \mathrm{a}$ & $3,13 \pm 0,45 \mathrm{~b}$ \\
Totale antenne & $56,66 \pm 5,86 \mathrm{a}$ & $49,17 \pm 4,81 \mathrm{~b}$ \\
\hline
\end{tabular}

A1 à A9: Articles du flagelle. Les valeurs moyennes portant les mêmes lettres sur une même ligne ne sont pas significativement différentes $(p>0,05)$.

Tableau 4 : Morphométrie des pattes des adultes de Diastocera trifasciata

\begin{tabular}{|c|c|c|}
\hline \multirow{2}{*}{ Structure (mm) } & \multicolumn{2}{|c|}{ Moyenne \pm écart-type } \\
\hline & Mâle & Femelle \\
\hline Patte antérieure & $34,73 \pm 3,40 a$ & $34,32 \pm 3,24 a$ \\
\hline Coxa & $4,40 \pm 0,57$ & $4,29 \pm 0,54$ \\
\hline Trochanter & $2,21 \pm 0,33$ & $2,16 \pm 0,37$ \\
\hline Fémur & $11,25 \pm 1,33$ & $10,98 \pm 1,12$ \\
\hline Tibia & $12,21 \pm 2,16$ & $12,38 \pm 1,27$ \\
\hline Tarses & $5,17 \pm 0,68$ & $5,15 \pm 0,65$ \\
\hline Griffes & $3,78 \pm 0,44$ & $3,67 \pm 0,43$ \\
\hline Patte médiane & $32,49 \pm 3,01 b$ & $32,13 \pm 2,87 b$ \\
\hline Coxa & $3,69 \pm 0,54$ & $3,51 \pm 0,49$ \\
\hline Trochanter & $2,12 \pm 0,47$ & $2,18 \pm 0,35$ \\
\hline Fémur & $10,52 \pm 1,18$ & $10,47 \pm 1,07$ \\
\hline Tibia & $11,18 \pm 1,16$ & $11,15 \pm 1,15$ \\
\hline Tarses & $5,35 \pm 0,65$ & $5,27 \pm 0,62$ \\
\hline Griffes & $3,68 \pm 0,43$ & $3,72 \pm 0,49$ \\
\hline Patte postérieure & $31,03 \pm 2,92 \mathrm{c}$ & $30,83 \pm 2,53 \mathrm{c}$ \\
\hline Coxa & $6,12 \pm 0,72$ & $6,31 \pm 0,69$ \\
\hline Trochanter & $2,32 \pm 0,49$ & $2,30 \pm 0,35$ \\
\hline Fémur & $11,07 \pm 1,07$ & $10,90 \pm 0,93$ \\
\hline Tibia & $10,65 \pm 0,99$ & $10,61 \pm 0,96$ \\
\hline Tarses & $5,25 \pm 0,54$ & $5,25 \pm 0,64$ \\
\hline Griffes & $3,64 \pm 0,49$ & $3,55 \pm 0,52$ \\
\hline
\end{tabular}

Les valeurs moyennes portant les mêmes lettres sur une même ligne ne sont pas significativement différentes $(p>0,05)$. Les valeurs moyennes portant les lettres différentes dans une même colonne sont significativement différentes $(p>0,05)$.

\section{DISCUSSION}

La présente étude a permis de décrire la morphologie de Diastocera trifasciata et de déterminer les mensurations des différentes parties du corps du mâle et de la femelle. Cette espèce se distingue des autres Cerambycidae par ses élytres à quatre bandes noires alternées de trois bandes jaunes. Cette discrimination à partir de l'ornementation de ses élytres a été mentionnée par Brunck et Fabre (1970) ainsi que Lefèbvre et al. (1973). Ils indiquent que les élytres $D$. trifasciata présentent trois larges bandes transverses 
orangées ou jaune séparées par deux bandes transverses noires plus ou moins irrégulières, avec la base et l'extrémité toujours noires. Cette étude a révélé que les adultes de $D$. trifasciata ont une longueur du corps qui varie entre 30,21 et $49,66 \mathrm{~mm}$ de long et une largeur qui se situe entre 9,10 et $16,13 \mathrm{~mm}$. Ces résultats rejoignent ceux de Brunck et Fabre (1970) qui ont rapporté que cet insecte a une longueur comprise entre 33 et $48 \mathrm{~mm}$ et une largeur entre 12 et $16 \mathrm{~mm}$. De même, les femelles $(41,40 \pm 3,19 \mathrm{~mm})$ sont plus grandes que les mâles $(40,17 \mathrm{~mm})$ mais, cette différence n'étant pas significative, la longueur du corps ne permet pas de distinguer les sexes chez $D$. trifasciata. En effet, plusieurs auteurs ont relaté que chez les Cerambycidae, il n'est pas conseillé d'utiliser la taille du corps des adultes pour la distinction des sexes (Wilcken et al., 2002; Galleja, 2004). Par ailleurs, chez $D$. trifasciata, les antennes sont toujours plus longues que le reste du corps. Chez le mâle, elles sont 1,41 fois plus longues que le corps tandis que chez les femelles, elles sont 1,18 fois plus longues. Ces résultats corroborent ceux de Akessé et Ouali-N'Goran (2018) qui ont rapporté que le dimorphisme sexuel chez $D$. trifasciata est marqué par des antennes plus longues chez les mâles que chez les femelles. La différence de la longueur des antennes chez les Cerambycidae semble être fréquente chez plusieurs espèces, surtout chez celles appartenant au genre Oncideres (Lingafelter et Hoebeke, 2002 ; Seffrin et al., 2006). Divers auteurs affirment que cette différence est liée à la reproduction qui est la phase la plus importante de la

\section{CONCLUSION}

La détermination des caractères morphométriques des adultes de Diastocera trifasciata et les comparaisons entre mâles et femelles a permis de relever que $D$. trifasciata est un insecte au corps robuste très sclérifié. II mesure en moyenne $40,79 \pm 3,28 \mathrm{~mm}$ de long et $13,96 \pm 1,52 \mathrm{~mm}$ de large. II se caractérise principalement par ses élytres à quatre bandes noires et trois bandes jaunes ; avec la base et l'extrémité des élytres toujours noires. En outre, la longueur des

\section{REMERCIEMENTS}

Les auteurs expriment leur gratitude à tous les producteurs qui ont bien voulu céder leur verger pour les captures des insectes. Ils remercient également la banque mondiale et le centre d'Excellence Africain sur vie des Cerambycidae (Paulino-Neto, 2016). Lopes et al. (2005) ont rapporté que les mâles de l'espèce Phoracantha simipunctata (Coleoptera : Cerambycidae) utilisent leurs longues antennes pour se mettre en contact avec les femelles. La grande taille des antennes des mâles leur offre plus de faciliter pour trouver une partenaire sexuelle, par contre les antennes des femelles sont courtes car elles n'ont aucun rôle sexuel (Hanks et al., 1997). En général, les coléoptères mâles sont dotés d'antennes plus longues et plus élaborées que les femelles, afin d'augmenter la surface disponible pour la détection de la phéromone sexuelle émanant des femelles (Hanks et al., 1997 ; Lemes et al., 2014). Les femelles se distinguent également des mâles par la taille et la structure du dernier sternite abdominal. Cette même observation a été faite par Brunck et Fabre (1970) ainsi que Lefèbvre et al. (1973) qui ont mentionné que les femelles de $D$. trifasciata diffèrent des mâles par un dernier sternite plus long et marqué par un sillon longitudinal. Selon Asogwa et al. (2009), le dernier sternite est un caractère utilisé pour différencier le mâle de la femelle de D. trifasciata. Lopes et al. (2015), indiquent que le dernier sternite abdominal est l'un des traits biométriques qui permet de faire la distinction des sexes chez Psacothea hilaris hilaris (Pascoe) (Coleoptera : Cerambycidae: Lamiinae). Ces auteurs rajoutent que la combinaison de l'observation de ce trait et de la longueur des antennes permettent de préciser les sexes chez les Cerambycidae.

antennes est plus importante que celle du corps quel que soit le sexe. II ressort de cette étude que chez $D$. trifasciata, il existe un dimorphisme sexuel marqué par des antennes plus longues chez le mâle et le dernier sternite abdominal femelle marqué par un sillon longitudinal. La connaissance de tous ces caractères de ce grand ravageur de l'anacardier permettra d'envisager une stratégie de lutte ciblée plus efficiente.

les Changements Climatiques, la Biodiversité et l'Agriculture Durable (CEA-CCBAD) de l'université Félix HOUPHOUÊT-BOIGNY pour le financement des travaux. 


\section{REFERENCES}

Adeigbe 00, Olasupo FO, Adewale BD, Muyiwa AA, 2015. A review of cashew research and production in Nigeria in the last four decades. Science Research Essays 10(5), 196-209.

Akessé EN, Ouali-N'goran SWM, 2018. Reproductive Parameters of Diastocera trifasciata (Fabricius, 1775) (Coleoptera: Cerambycidae: Lamiinae), Cashew Branches Girdler in Côte d'Ivoire, Under Semi-Natural Conditions. International Journal of Scientific and Research Publications. 8(9), 686-698.

Akessé EN, Ouali-N'goran SWM, N'Dépo OR, Koné T, Koné D, 2015. Prospective Study of the Insect Fauna Associated with Anacardium occidentale L. (Salpindales: Anacardiaceae) in Five Producing Areas of Côte d'Ivoire," In Proceedings on the Third International Cashew Conference: Cashew for Health Wealth and Environment. Held at Serena Hotel, Dar Es Salaam, Tanzania, 164-172.

Akessé EN, Ouali-N'Goran SWM, N'Dépo OR, Tano DKC, 2018. Population fluctuation of Diastocera trifasciata (Fabricius, 1775) (Coleoptera: Cerambycidae), cashew branches girdler in the Brobo locality (Central Côte d'lvoire). Journal of Entomology and Zoology Studies 6(5), 1064-1070.

Asogwa EU, Anikwe JC, Ndubuaku TCN, Okelana FA, Hammed LA, 2009. Host plant range and morphometrics descriptions of an emerging insect pest of cashew, Plocaederus ferrugineus. (Coleoptera: Cerambycidae) in Nigeria: a preliminary report. International Journal of Sustain Crop Production 4(3): 2732.

Brunck F, Fabre JP, 1970. Note sur Analeptes trifasciata Fabricius, Coléoptère Cérambycidé, grave ravageur d'Anacardium occidentale en Côte d'Ivoire. Bois et Forêts des Tropiques $134: 15-19$.

CCA, 2016. Atelier d'élaboration du programme national sur la recherche de l'anacardier (PNRA) 2016-2026, rapport $7 \mathrm{p}$.

Diop M, 2016. Côte d'Ivoire : Premier producteur mondial de noix de cajou, rapport $1 \mathrm{p}$.

Djaha AJB, N'Da AA, Dosso M, Kouakou CK, Djidji AH, Minhibo MY, Kpokpa H, Bambio ZK, Bambara $J, 2017$. Bien produire des plants greffés d'anacardier en Côte d'Ivoire, 4 p.
FAO, 2005. Côte d'Ivoire irrigation en Afrique en chiffres. AQUASTAT, $10 \mathrm{p}$.

Galleja AG, 2004. Dimorfismo sexual en imagos del coleóptero cerambicido Xylotrechus arvicola Olivier. Boletín de Sanidad Vegetal Plagas 30(1): 19-24.

Hammed LA, Anikwe JC, Adedeji AR, 2008. Cashew Nuts and Production Development in Nigeria. American-Eurasian Journal of Scientific Research 3(1): 54-61.

Hanks LM, Millar JG, Paine TD, 1997. Mating behavior of the eucalyptus longhorned borer (Coleoptera: Cerambycidae) and the adaptive significance of the longhorned. Journal of Insect Behavior 9(3) : 383-439.

Koné M, 2010. Analyse de la chaîne de valeur du secteur anacarde de la Côte d'Ivoire. Initiative du Cajou Africain, 76 p.

Kra KD, Kwadjo KE, Douan BG, Kouamé KL, Ouattara KV, Doumbia M, 2017. Evaluation des dégâts d'Analeptes trifasciata sur les anacardiers dans les régions du Béré et de l'Iffou (Côte d'Ivoire). Journal of Applied Biosciences 112: 10969-10977.

Lebailly P, Lynn S, Seri H, 2012. Etude pour la préparation d'une stratégie pour le développement de la filière anacarde en Côte d'Ivoire. Proposition d'une stratégie pour le développement de la filière anacarde, $47 \mathrm{p}$.

Lefèbvre A, Leturcq PH, Praloran JC, 1973. Etudes sur l'anacardier: Techniques de création et d'entretien des plantations fruitières d'anacardier. Fruits 28(4): 271-278.

Lemes PG, Dos Anjos N, Jorge IR, Cordeiro G, 2014. Sexual dimorphism in the twig girdler Oncideres ocularis Thomson (Coleoptera: Cerambycidae). Entomological News 123(5): 358-364.

Lingafelter SW, Hoebeke ER, 2002. Revision of the genus Anoplophora (Coleoptera: Cerambycidae). Entomological Society of Washington, Washington DC, USA.

Lopes O, Marques PC, Araújo J, 2005. The role of antennae in mate recognition in Phoracantha semipunctata (Coleoptera: Cerambycidae). Journal of Insect Behavior 18(2): 243-257.

Lupi D, Jucker C, Rocco A, Harrison R, Colombo M, 2015. Notes on biometric variability in invasive species: the case of Psacothea hilaris hilaris. Bulletin of Insectology 68 (1): 135-145. 
N'Dépo OR, Chérif $M$, Johnson $F$, Kassi KFJM, N'Guessan AC, Silué N, Akessé EN, Koné D, N'Goran OM, 2017. Inventaire des insectes ravageurs du verger anacardier dans les régions de Bounkani, Gontougo et IndénieDjablin au Nord-Est en Côte-d'Ivoire. Afrique Sciences 13: 333-343.

Paulino-Neto HF, 2016. A Review of the Biology and Ecological Interactions of Oncideres (Cerambycidae): Brazilian Wood Borers Species. Journal of Ecosystem and Ecography 6: 223

Seffrin RCAS, Costa EC, Couto MRM, Lopes SJ, 2006. Medidas morfométricas de fêmeas e machos de Oncideres dejeani Thompson, 1868. Ciência Rural 36(4): 1313-1316.

Viana FMP, Cardoso JE, Suraiva HAO, 2007. First report of a bacterial leaf and fruit spot of cashew nut (Anacardium occidentale) caused by Xanthomonas campestris pv, Mangifera indica in Brazil. Plant Disease 91: 13-61.

Wilcken CF, Berti EF, Ottati ALT, Firmino DC, Couto EB, 2002. Ocorrência de Phoracantha recurva Newman (Coleoptera: Cerambycidae) em eucalipto no Estado de São Paulo, Brasil. Scientia Forestalis 62 : 149-153. 